\title{
Beyond Motor Imagery for Cognitive Choreography
}

Martina Morasso, Pietro Morasso

Choreography, as the art of creating and arranging dances, draws its name from two Greek words: dance and write. According to mythological tradition, the choreutic art was under protection of Terpsichore, one of the nine muses who were the daughters of Zeus and Mnemosine, the goddess of memory. Moreover, dance, as an artistic form of movement, was strongly linked to music (typically, icons of Terpsichore hold a musical instrument) as well as to poetry or story telling that were indeed a shared interest of all the sister-muses. Although dance and music are both performing arts which seem to vanish after a performance, the emphasis on memory, given by the name itself of the mother of arts, and the prescription of writing, inherent in the notion of dance-art (choreography), both highlight the need to associate the kinetics of the body with the flow of the mind that is the inner and hidden source of it.

In a general sense this is the fundamental reason for investigating the multi-faceted points of contacts between cognition and choreography, namely between neuroscience and performing arts. In a more specific sense one could focus on the challenge faced by choreography as a language for representing written record of dances, or dance notation, in analogy with the techniques invented for music notation.

\section{Pros and Cons of Dance Notation}

Many systems of dance notation have been invented, starting with the Beauchamp-Feuillet notation used for Baroque dance, which was organized along the path on the floor to be followed by the dancers. More recent systems have been developed in the twentieth century for movement notation in general, namely for recording and analyzing human movement in specific areas of 
action, including dance. Labanotation or Kinetography Laban is the oldest one, developed by Rudolf Laban in the 1930's (Laban 1926): it uses abstract symbols to define different aspects of movements (Direction, Duration, Dynamic quality, Body part). The Benesh movement notation or choreology was developed by Joan and Rudolf Benesh in the late 1940's: it is based on a more visual form of notation and it uses five lines, representing the dancer's position as viewed from behind. The Eshkol-Wachman system was developed by Noa Eshkol and Abraham Wachman in the late 1950's: the body is represented as a stick figure and the position of those segments in three-dimensional space is characterized by a spherical coordinate system.

The pragmatic problem with such systems of dance notation is that few choreographers and even fewer dancers are literate in them. As currently practiced, dance notation is basically used only for recording, rather than creating and learning. These systems are rather static and with a poor 3D expressiveness; moreover, although they are focused on movements, they can only provide an approximated representation of movement. In a sense, the bottleneck, which has been encountered by all such methods, lies in the inadequacies of the medium: the piece of paper, which is quite adequate for hosting a novel or a music score, is obviously inadequate to support the kind of multidimensionality which characterizes motor performance. We may suggest that the multidimensionality of dance is similar to the structure of a Japanese garden, which can hide in a limited space an infinity of landscapes, as seen by different viewers. In contrast, the modern staff notation, which is used by musicians of many different genres throughout the world, has succeeded because it captures the basic elements of a musical composition: the staff acts as a framework upon which pitches are indicated by placing oval noteheads on the staff lines or between the lines. Moreover, the pitch of the oval musical noteheads can be modified by accidentals and the duration is shown with different note values (stemless hollow oval, hollow rectangle etc.). Presently, the shaky tradition of movement notation is even challenged by new technological possibilities for the visual and digital representation of movement, including computer graphics and motion capture systems, capable to store the rotation patterns of all the joints and the trajectories in threedimensional space of the different body parts. However, the use of digital technologies may come with a price-tag: the risk of hiding the (choreutic) message behind an attractive but distractive technological envelope without 
succeeding to solve the basic problem of developing an appropriate language of movement understanding and dance creating.

On the other hand we should also take into account that, well aware of the limitations of the movement notation system that was tagged with his name, Rudolf Laban focused as well on holistic methods for the analysis of movement (Laban Movement Analysis) that include four main external aspects (Body, Space, Time, Shape) as well as an internal element characterized as Effort. In any case, Laban himself stressed that he had no method and had no wish to be presented as having one: he served as a guide for his students, suggesting ideas that they should work from, but he did not explicitly direct his students, neither did he tell them how to perform, nor what quality to adopt while performing. This kind of approach is well expressed by one of his best known students, Mary Wigman: "Laban had the extraordinary quality of setting you free artistically, enabling you to find your own roots, and thus stabilized, to discover your own potentialities, to develop your own technique and your individual style of dancing." (Wigman 1973: 35) Rather a vivid spirit of enquiry is the main legacy associated with global metaphors formulated by Laban like the Icosahedron as the scaffolding of the kinesphere, related to a view of human movement as a continuous creation of fragments of crystalline forms. It is also worth mentioning the link of the thinking of Laban, choreographer and movement scientist, with two other contemporaries of him, active between the two world wars: the Russian neuroscientist Nikolai A. Bernstein and the American neuropsychologist Karl S. Lashley. The general view that became clear later on, after the independent investigations of such scientists, was that coordinating the complexity of actions, what can also be called Synergy Formation, the underlying control system has to deal with two typically contrasting requirements: the need to choose "one" from infinite possible solutions: Bernstein's Degrees of Freedom Problem and the need to choose "one solution" from an infinite palette of possible ways: Lashley's Principle of Motor Equivalence. It is the incommensurable complexity of synergy formation, in comparison with harmonic-melodic complexity of music, that does not allow any chance of success to dance notation methods focused on the coordination of all the degrees of freedom. 


\section{Dance and Cognition}

What is the relevance of cognitive science for choreography, as an artistic, creative activity? This is a question that has been formulated by many people (Hagendoorn 2003; DeLahunta 2004; Morasso/Morasso 2017). The main issue, in order to escape from generic talk, is that cognitive science covers a very large area of topics from neuroscience to cybernetics. And for mainstream cognitive science, that focuses on the mind as an information processing system, the body is an extraneous bunch of bones and muscles separated from the mind. For a choreographer and a dancer the body is not only the medium but the message as well. More specifically, we are convinced that the type of cognition that is relevant for dance is Embodied Cognition. In short terms this implies the recognition of the deep dynamic unity and interaction between brain, body, mind, and environment. This is a general attitude that is at the same time monistic and dualistic and agrees as well with the general principles of biological autonomy advocated by Varela (Varela 1979) in the framework of enactivist theories. This implies the concept that the essence of cognition is the capacity to produce "smart and beautiful" actions, i.e. actions that are goal-directed and shaped by prospection (von Hoftsten 2008), following the natural tendency to continually predict the likely outcome of an action in order to update its execution for matching the final goal with the current path followed to reach it. The main idea is that our body, in all its aspects (sensory, motor, and body-environment interaction), shapes and organizes our mind, including high-level features (like memory, concepts, and categories) and abstract tasks (like reasoning, judgment, and previewing). This unitary formulation of the body-mind system is clearly opposed to various forms of dualism, from the old-fashioned Cartesian dualism to more recent theories like cognitivism and conventional artificial intelligence. In particular, embodied cognition is characterized by several features:

1. it is situated, in the sense that it is an online process, which takes place in the context of task-relevant sensorimotor information;

2. it is time pressured, i.e., it is constrained by the requirements of real-time interaction with the environment;

3. the environment is part of the cognitive system;

4. it is intrinsically action oriented and even "off-line cognition", namely cognition without overt action, is bodily based. 


\section{From Motor Imagery to Motor Control: The Peter Pan's Shadow Metaphor}

There is no doubt that motor imagery, as a mental process by which an individual rehearses a given action by simulating it mentally, is relevant for dancers. In sport training it has been adopted as an effective technique of mental practice of action; in neurological rehabilitation it may help stroke patients to recover some degree of volitional control of movement; in dance it has been used for supporting the imagination of actions shaped by external virtual objects. The underlying neurophysiological evidence is the strong similarity of brain activation patterns that characterize real (overt) movements and imagined (covert) movements. However, the link between overt and covert movements is much stronger. As a matter of fact, overt actions are a small portion of human movements: the great majority is covert and fulfills a multiplicity of functions such as anticipation, prediction, and organization of the flow of movements driven by a goal. A crucial contribution to this embodied cognitive framework is the Mental Simulation Theory by (Jeannerod 2001): it posits that cognitive motor processes such as motor imagery, movement observation, action planning, and verbalization share the same representations with motor execution and are implemented by running or animating an internal model of the Body Schema, in other words a kind of internal cinema where the brain is the puppeteer that pulls the strings of the animated body.

In a previous work (Morasso/Morasso 2017) a computational metaphor was proposed that is derived from the Mental Simulation Theory, namely the Peter Pan's Shadow Metaphor: Peter Pan's Shadow is a visualization of the Body Schema and its conscious animation is the engine for linking body and mind in dance performance. This is a view about synergy formation in motor control that has the purpose of answering the challenge posed by the previously mentioned Degrees of Freedom Problem: it is inspired by the Equilibrium Point Hypothesis (Feldman 1966) and, in particular, by its extension for including both overt and covert movements called Passive Motion Paradigm (Mohan/Bhat/Morasso 2019). The general idea of the PMP computational metaphor is that the multiple dynamic interactions that characterize any given task can be implemented as multiple superimposed force fields that collectively shape an energy function, whose equilibrium point drives the synergy formation process, coordinating the different body parts participating 
to a task as a single integrated organism. These views about motor control and synergy formation are based on the concept that in order to understand whole body motion it is convenient to shift the emphasis from movement to the forces that drive it: movement is the consequence while force is the cause. What is gained by the brain in this manner is a drastic simplification of the control variables in analogy with the simplification of the task of a puppeteer who needs to pull a limited number of strings for animating a marionette. The modeling framework logically implied by this concept comprises a bodyschema, as an internal model of the whole body, and a synergy formation mechanism that animates the Body Schema: Peter Pan and his shadow, i.e. the animated Body Schema.

This metaphor, in our view, captures the unitary nature of overt (real) and covert (imagined) actions: overt actions are just the tip of an iceberg, in the sense that they are the final result of a cognitive process of mental simulations aimed at testing the feasibility of potential actions, foreseeing the potential consequences in relation to oneself and others (in social contexts), composing strings of potential actions or recycling already experienced strings. The grounding for this open question is an emerging trend from motor neurosciences suggesting that "generation, simulation and understanding" of actions consistently engage a shared cortical network in the motor areas of the brain and overt movement is just the tip of the iceberg, recycled to enable the diversity of covert actions without movements.

\section{Beyond Movement Notation in Choreography: From Embodied Cognition to Cognitive Choreography}

In alternative to movement notation a completely different approach is exemplified by Tai Chi Chuan: although it deals with body movements, it is mainly focused on force in different senses. As a martial art, Tai Chi Chuan is the study of appropriate change in response to outside physical forces, the study of yielding and sticking to an incoming attack rather than attempting to meet it with opposing force; at a more abstract, cognitive level Tai Chi Chuan is a way to work directly with one's qi, or life force. Moreover, Tai Chi Chuan sequences (or forms) can be explained and memorized in a metaphorical manner by using action images, like "White Crane Spreads Its Wings", 
"Grasp Sparrow's Tail" or "Wave Hands Like Clouds", that imply a physical interaction, namely an exchange of force, with an external object.

The idea that motor imagery is a powerful tool for understanding and learning action sequences (Overby/Dunn 2011) is also present, specifically in the field of choreography, in the work with dance imagery initiated by Todd (1937) that later became known as Ideokinesis (Sweigard 1978). The Improvisation Technologies described by William Forsythe (Forsythe 1997) are another way to explode the Laban Kinesphere. According to this approach, the focus of the dancer's action must be on the beginning rather than the end of a particular action. The initial state of mind incorporates a whole world of possibilities from which the specific unfolding of the action can take place as a consequence of a number of "distorting" force fields, in such a way to match/ avoid/intrude/extrude points, lines, volumes of an expanding kinesphere.

The Peter Pan's shadow metaphor is quite compatible with the Tai Chi Chuan mindset. The Peter Pan's shadow is the action image, recalled from memory before carrying out the action, and the virtual forces springing out of the image are the driving agents for the actual movements performed by "Peter's body". The power and efficiency of this force-based language for expressing action sequences is demonstrated by the fact that million people in the world do not have difficulty to learn, memorize, and execute Tai Chi Chuan sequences (or forms) in contrast with the different movement notation systems. Again, we stress the point that the emphasis is not and must not be on the final shape of an action but on the initial visualizations, expectations, internal simulations that initiate and feed the action flow. Thus, a smart movement will naturally take advantage of the affordances (in the sense of J. J. Gibson) made available during the course of an action, naturally matching the forces at play in the unfolding environment. In this way, it is possible, on one side, to escape the rigidity of a predefined choreographic script and, on the other, to avoid the infinite but chaotic variability of unconstrained movements. Peter Pan's shadow in a sense operates as a kind of Gestalt capable to attract the action to specific shapes but flexible enough to avoid them or escape from them if unexpected events occur. The dynamic interaction between Peter Pan's shadow and Peter Pan's body can achieve a balance between stabilization and destabilization.

The issue here is that choreography, as well as embodied cognition, is about composition of action sequences, and compositionality of action primitives is easier and more natural in terms of (a small set of) forces/drives than 
of (a large set of elementary) movements. In summary, we suggest that by bringing together the Peter Pan's Shadow metaphor and the Tai Chi Chuan mindset we can produce a new approach to choreography, grounded on solid principles of embodied cognition.

\section{References}

DeLahunta, Scott (2004): Separate Spaces: some cognitive dimensions of movement. Species of Spaces (a DIFFUSION eBook Series), London: Proboscis.

Feldman, Anatol G. (1966): On the functional tuning of the nervous system in movement control or preservation of stationary pose. II. Adjustable parameters in muscles (article in Russian), in: Biofizika, Vol. 11 No. 3, pp. 498-508.

Forsythe, William (1997): Improvisation Technologies: A Tool for the Analytical Dance Eye, Ostfildern: Hatje Cantz.

Hagendoorn, Ivar (2003): Cognitive Dance Improvisation: How Study of the Motor System Can Inspire Dance (and Vice Versa), in: Leonardo, Vol. 36 No. 3, pp. 221-227.

Jeannerod, Marc (2001): Neural simulation of action: a unifying mechanism for motor cognition, in: NeuroImage, Vol. 14, pp. S103-S109.

Laban, Rudolf (1926): Choreographie: Erstes Heft. Jena: Eugen Diederichs.

Mohan, Vishwanatan/Bhat, Ajaz A./Morasso, Pietro. (2019): Muscleless Motor synergies and actions without movements: From Motor neuroscience to cognitive robotics, in: Physics of Life Reviews, Vol. 30, pp. 89-111.

Morasso, Pietro/Morasso, Martina (2017): From Embodied Cognition to Peter Pan's Shadow as a Metaphor for Cognitive Choreography. Brain, Body, Cognition, in: Nova Science Publishers, Vol. 7 No. 1, pp. 1-8.

Overby, Lynette Y./Dunn, Jan (2011): The History and Research of Dance Imagery: Implications for Teachers, in: IADMS Bulletin for Teachers, Vol. 3 No.2, pp. 9-11.

Sweigard, Lulu (1978): Human Movement Potential: Its Ideokinetic Facilitation, New York: Dodd Mead \& Company.

Todd, Mabel E. (1937): The thinking body, New York: Paul B. Hoeber Inc. Reprint of 1937 Edition in 2017, Gouldsboro ME: Gestalt Journal Press.

Wigman, Mary (1973) The Mary Wigman book: Her Writings, Middletown: Wesleyan University Press. 